\title{
Entropic Splitter for Particle Separation
}

\author{
D. Reguera, ${ }^{1, *}$ A. Luque, ${ }^{1}$ P. S. Burada,${ }^{2}$ G. Schmid,${ }^{3}$ J. M. Rubí, ${ }^{1}$ and P. Hänggi ${ }^{3}$ \\ ${ }^{1}$ Departament de Física Fonamental, Facultat de Física, Universitat de Barcelona, Martí i Franquès 1, \\ E-08028 Barcelona, Spain \\ ${ }^{2}$ Max-Planck Institut für Physik komplexer Systeme, Nöthnitzer Str. 38, D-01187 Dresden, Germany \\ ${ }^{3}$ Institut für Physik, Universität Augsburg, Universitätsstrasse 1, D-86135 Augsburg, Germany
}

(Received 9 September 2011; published 13 January 2012)

\begin{abstract}
We present a particle separation mechanism which induces the motion of particles of different sizes in opposite directions. The mechanism is based on the combined action of a driving force and an entropic rectification of the Brownian fluctuations caused by the asymmetric form of the channel along which particles proceed. The entropic splitting effect shown could be controlled upon variation of the geometrical parameters of the channel and could be implemented in narrow channels and microfluidic devices.
\end{abstract}

DOI: 10.1103/PhysRevLett.108.020604

Matter very often manifests itself as divided into very fine parts whose nature dictates the overall properties of the system. Grains, conglomerates, or mesostructures dispersed in a liquid or liquidlike phase are basic structures frequently found in many physicochemical and biological problems. The dissimilarity of these units, resulting from random fractionation or self-assembling processes giving rise to a very disparate distribution of sizes, originates a heterogeneous response of the system which makes its typification difficult. To obtain pure substances by separating wanted from unwanted elements presents a major challenge in basic research and industrial processing, and in nanotechnology as well.

Particle separation techniques use the fact that the response of the particles to external stimulus, such as gradients or fields, depends on their size. Filtering particles of different size is traditionally performed by means of centrifugal fractionation [1], phoretic forces [2-4], or external fields [5]. By means of these methods, the sorting of particles proceeds either by size exclusion, as happens in a sieve, or by migration through the host medium, a gel or porous media. In these cases all particles move in the same direction but at different speeds. Novel separation techniques based on flashing [6-8], drift [9], "deterministic" $[10]$, and geometric $[11,12]$ ratchets have also been proposed for sorting [13].

Here we present a novel splitting mechanism that separates particles in different directions by purely entropic means. The working principle relies on the combined action of a static force and an entropic rectification [14-17]. Small particles follow the force, whereas the motion of big particles is rectified to proceed in the opposite direction resulting in a faster splitting. This mechanism could be readily implemented in microchannels or microfluidic systems. The geometry of the channel can be tuned to be very selective leading to an efficient separation of particles of very similar radii.
PACS numbers: 05.40. $-\mathrm{a}, 02.50 . \mathrm{Ey}, 05.10 . \mathrm{Gg}, 05.60 .-\mathrm{k}$

The Brownian motion of particles in confined geometries exhibits a very rich and striking phenomenology [18-23]. The effects of confinement can be described by means of an effective entropic potential resulting from the variation of the space accessible to particles along the transport direction $[18,24,25]$. The height of the entropic barrier associated with the bottlenecks depends on particle radius. In an asymmetric channel, the resulting entropic potential becomes asymmetric, and rectification of a zero-mean oscillating force can occur. The strength of this rectification depends on the particle radius and is stronger for large particles (for which the entropic barrier is larger, see Fig. 1). Thus, in the presence of a small static force directed in the opposite direction of rectification, it is possible to separate particles of different sizes. The

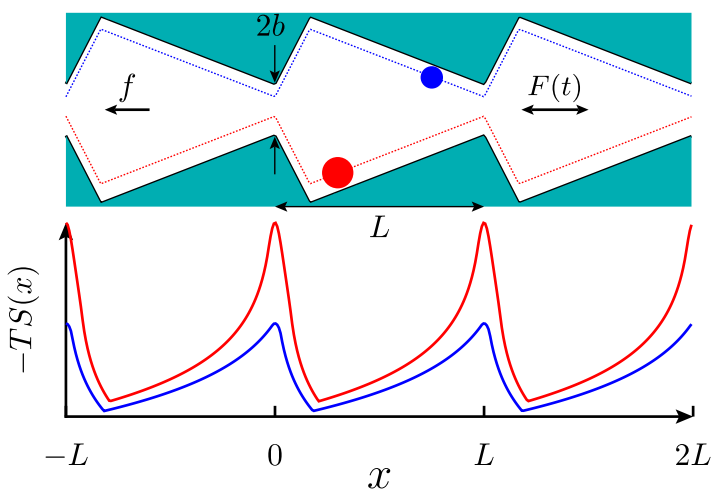

FIG. 1 (color online). (top) Schematic illustration of the twodimensional channel confining the motion of the Brownian particles. The structure is defined by Eq. (2). Brownian particles are driven by a constant force $\vec{f}$ and a square wave force $\vec{F}(t)$ along the longitudinal direction. The values of the chosen parameters defined in Eq. (2) are: $m_{1}=2, m_{2}=0.4, b / L=0.1$. The dashed lines represent the limit for the positions of the center of the particles within the channel. (bottom) Effective entropic potential for the two particle sizes depicted above. 
trajectories of small particles mainly follow the force, whereas large particles move in the opposite direction. To illustrate this effect we have chosen the geometry depicted in Fig. 1.

The dynamics of a Brownian particle in a 2D channel, as the one depicted in Fig. 1, driven by a static force $f$ and an oscillating (square wave) force $F(t)$, both applied along the principal axis of the channel, can be described by means of the Langevin equation which, in the overdamped limit, reads

$$
\gamma \frac{d \vec{r}}{d t}=-[f+F(t)] \vec{e}_{x}+\sqrt{\gamma k_{B} T} \vec{\xi}(t),
$$

where $\vec{r}$ denotes the position of the particle, $\gamma$ is the friction coefficient, $\vec{e}_{x}$ is the unit vector along $x$ direction, and $\vec{\xi}(t)$ is a Gaussian white noise with zero mean which obeys the fluctuation-dissipation relation $\left\langle\xi_{i}(t) \xi_{j}\left(t^{\prime}\right)\right\rangle=2 \delta_{i j} \delta\left(t-t^{\prime}\right)$ for $i, j=x, y$. The explicit form of the oscillating driving force is $F(t)=A \operatorname{sgn}[\sin (\Omega t)]$ where $A$ is the amplitude, $\operatorname{sgn}[t]$ represents the sign function, and $\Omega$ is the driving frequency. This choice does not represent a restriction on the particle splitting effect which could also be observed for a sinusoidal driving force.

The Langevin equation (1) must be solved by imposing vanishing outflow at the walls of the structure. For the 2D structure depicted in Fig. 1, the walls are defined by

$$
y_{u}(x)=\left\{\begin{array}{ll}
b+m_{1} \bar{x} & \text { if } \bar{x}<c \\
b+m_{2}(L-\bar{x}) & \text { otherwise }
\end{array},\right.
$$

where $y_{u}(x)$ and $y_{l}(x)=-y_{u}(x)$ correspond to the upper and lower boundary functions, respectively, $b$ is the half-width of the bottleneck, $m_{1}$ and $m_{2}$ are the slopes of the walls, $L$ is the periodicity of the channel, $c=$ $L m_{2} /\left(m_{1}+m_{2}\right)$ indicates the location of the point of maximum width, and $\bar{x}=x \bmod L$ is the modulo function (to create a periodic structure) cf. Fig. 1.

For a hard particle of radius $r$ inside the channel, the space available for its center is restricted by a distance $r$ from the walls, reading

$$
w_{u}(x)=\left\{\begin{array}{ll}
-\sqrt{r^{2}-\bar{x}^{2}}+b, & 0 \leq \bar{x}<o_{p} \\
b+m_{1} \bar{x}-r \sqrt{\left(1+m_{1}^{2}\right)}, & o_{p} \leq \bar{x}<c_{p} \\
b+m_{2}(L-\bar{x})-r \sqrt{\left(1+m_{2}^{2}\right)}, & c_{p} \leq \bar{x}<L_{p} \\
-\sqrt{r^{2}-(\bar{x}-L)^{2}}+b, & L_{p} \leq \bar{x}<L
\end{array},\right.
$$

where $o_{p}=r m_{1} / \sqrt{\left(1+m_{1}^{2}\right)}, L_{p}=L-r m_{2} / \sqrt{\left(1+m_{2}^{2}\right)}$, and $\quad c_{p}=c+r\left[\sqrt{\left(1+m_{1}^{2}\right)}-\sqrt{\left(1+m_{2}^{2}\right)}\right] /\left(m_{1}+m_{2}\right)$. The lower parallel curve is just $w_{l}(x)=-w_{u}(x)$. Consequently, $2 w(x)=w_{u}(x)-w_{1}(x)$ gives the local width of the structure accessible for the center of a hard particle of radius $r$. Assuming throughout a dilute particle density and a strong viscous, low Reynolds number dynamics, all relevant hydrodynamic wall-particle interactions are small and of repulsive character [26]. Effectively, this results in a slightly increased effective particle radius; i.e., $r_{\text {eff }} \gtrsim r$. Such hydrodynamic interactions thus result in somewhat larger average transport velocities, note Figs. 2(a) and 2(b) below.

Also the friction depends on size, and using Stokes' law as an approximation one obtains $\gamma=\gamma_{0} r / b$ and $D=D_{0} b / r$, where $\gamma_{0}$ and $D_{0}=k_{B} T / \gamma_{0}$ are the friction and diffusion coefficients of a particle of radius equal to the bottleneck half-width $b$. To mimic the particular case of DNA electrophoresis, we will consider that the forces depend linearly on the radius of the particles. Specifically, we set

$$
f=f_{0} r / b \quad \text { and } \quad A=F_{0} r / b \text {, }
$$

where $f_{0}$ and $F_{0}$ are the strengths of the static and periodic forces for a particle of radius $b$.

For the sake of a dimensionless description, we scale all variables using three characteristic parameters: the characteristic length $L$, energy $k_{B} T$, and diffusion coefficient $D_{0}$. Particularly, $\tilde{x}=x / L, \tilde{y}=y / L, \tilde{b}=b / L, \tilde{w}_{l}=$ $w_{l} / L=-\tilde{w}_{u}, \tilde{t}=t / \tau$, and $\tilde{\Omega}=\Omega \tau$, where $\tau=L^{2} / D_{0}$ is the characteristic diffusion time. The scaled forces are $\tilde{f}=$ $f L / k_{B} T$ and $\tilde{F}(\tilde{t})=F(t) L / k_{B} T$. In the following, we shall omit the tilde symbols. In dimensionless form the Langevin equation (1) reads:

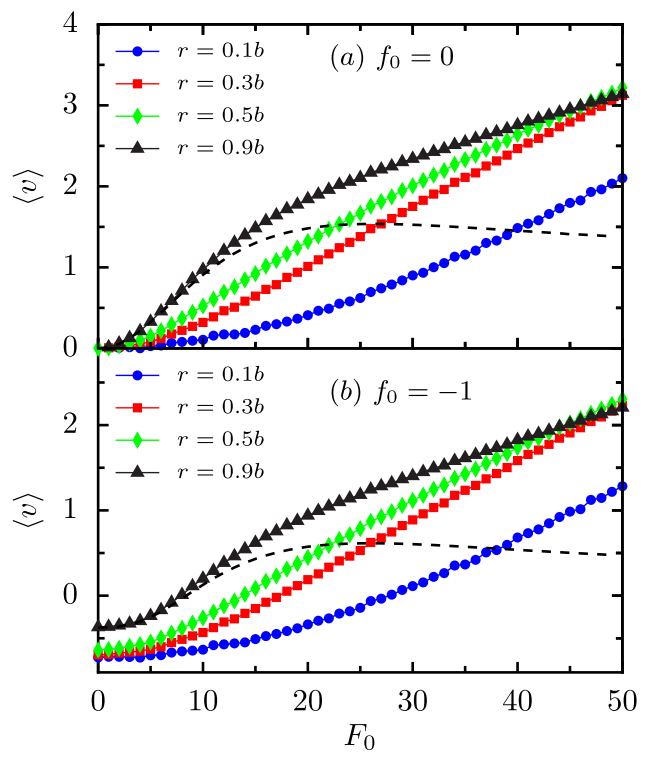

FIG. 2 (color online). Average current $v s$ the amplitude of the periodic forcing $F_{0}$ in the adiabatic limit $\left(\Omega=\Omega_{0}=\pi / 10\right)$ for particles of different radii, for a channel with $m_{1}=2, m_{2}=0.4$, and $b / L=0.1$. The solid lines indicate the results of the simulations, whereas the dashed line plots the prediction according to the FJ equation for $r=0.9 b$. For $f_{0}<0$ small particles go to the left, whereas big particles move to the right. The critical size that divides these two behaviors can be tuned by the value of $F_{0}$. 


$$
\frac{d \vec{r}}{d t}=-\left(f_{0}+F_{0} \operatorname{sgn}[\sin (\Omega t)]\right) \vec{e}_{x}+\sqrt{b / r} \vec{\xi}(t) .
$$

The description of this system alternatively can be given by the concept of an entropic potential [18] and the corresponding Fick-Jacobs (FJ) equation [18,19,24,25]

$$
\frac{\partial P(x, t)}{\partial t}=\frac{\partial}{\partial x}\left\{D(x)\left(\frac{\partial P}{\partial x}+V^{\prime}(x) P\right)\right\}
$$

where

$$
V(x)=U-T S(x)=[F(t)+f] x-\ln [2 w(x)],
$$

is the free energy including an entropic contribution $T S(x)=\ln [2 w(x)], \quad D(x)=b /\left\{r\left[1+w^{\prime}(x)^{2}\right]^{1 / 3}\right\}$ is the position-dependent diffusion coefficient, and the prime refers to the derivative with respect to $x$. This approximation is expected to be very accurate for bias strengths $\left|f_{0}\right|<1$, amplitudes $F_{0}<1$, and for $w^{\prime}(x)^{2} \ll 1$ [21].

In the adiabatic limit, the average velocity can be calculated as

$$
\langle v\rangle=\frac{J\left(F_{0}\right)+J\left(-F_{0}\right)}{2},
$$

where the current $J\left(F_{0}\right)$ is given by Stratonovich's formula [27-29];

$$
J\left(F_{0}\right)=\frac{1-e^{-\left(F_{0}+f_{0}\right) r / b}}{\int_{x_{0}}^{x_{0}+1} d z \frac{1}{D(z)} e^{V(z)} \int_{z-1}^{z} d x e^{-V(x)}} .
$$

Note that for very large driving strengths $F_{0} \gg 1$, while keeping a finite bias strength $\left|f_{0}\right|$, i.e., far beyond the regime of validity of the Fick-Jacobs approximation, the average velocity approaches the deterministic behavior $[14,19]$. In this limit we then find that $J\left(F_{0}\right) \approx-J\left(-F_{0}\right)$ so that an asymptotic vanishing net velocity $\langle v\rangle$ emerges. Note that this asymptotic regime of very large amplitude strength $F_{0}$ is not yet reached in Fig. 2.

Figure 2 plots the average current $v s$ the amplitude of the periodic forcing $F_{0}$, in the adiabatic limit $(\Omega=\pi / 10)$, for particles of different radii. In absence of static forcing, this asymmetric channel rectifies the oscillating force giving rise to a net positive velocity; i.e., all particles move towards the right [see Fig. 2(a)]. Its magnitude depends on the strength of the rectification which is more intense the larger the particle radius. If we apply a small static force in the negative direction [see Fig. 2(b)], for intermediate periodic forcing strengths $F_{0}$, particles larger than a given threshold radius move to the right, whereas particles smaller than that move to the left. In this way, one can separate particles of different radii and make them move in opposite directions. The splitting effect is illustrated schematically with Fig. 3.

Figure 2 also represents the results for the average velocity obtained from the FJ equation for the case $r=0.9 b$. The agreement with the simulation results is

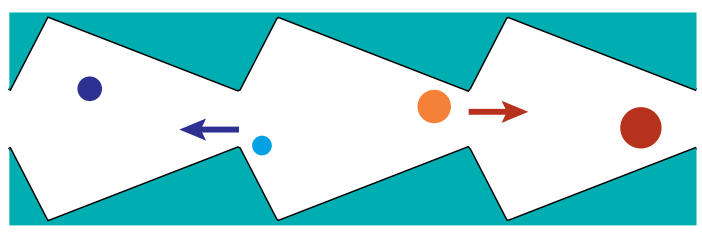

FIG. 3 (color online). Schematic illustration of the functioning of the entropic splitter. A mixture of particles of different radii is placed initially in the center. Under the combined presence of the static and periodic forcing, large particles move towards the right, whereas small particles follow the bias, i.e., are moving towards the left.

very good at small values of the force (for $F_{0}<10$ ). At larger values of $F_{0}$, the agreement is better when the radius increases or equivalently when entropic effects are more pronounced. In the domain of validity of the FJ description the corresponding applied forces already yield a significant entropic splitting.

The splitting effect can be tuned by the value of either the amplitude of the periodic forcing or the static force. Figure 4 represents the average value of the velocity $v s f_{0}$ for the same geometry and $F_{0}=20$. One can see that by tuning $f_{0}$, one can control the separation of particles of different sizes. For instance, by selecting $f_{0}=-1.5$, small particles of radius $r=0.1 b$ will move to the left with a velocity -0.7 , whereas large particles of radius $r=0.9 b$ will drift to the right with velocity 0.5 . More importantly, the velocity depends almost linearly on $f_{0}$, thus facilitating an efficient control of the separation effect. In addition, by progressively changing $f_{0}$, one obtains a device that, with a fixed geometry, can be used to continuously separate particles of any size.

We have also analyzed the effect of the frequency of the periodic forcing on the particles current. For small frequencies the results agree with those obtained in the adiabatic limit. As the frequency increases, the velocity becomes progressively smaller. Eventually, at very high frequencies, the change in the oscillating force is so fast that the particle cannot follow it and as a result there is a vanishing effect of the oscillating force.

The fact that particles of different sizes travel in opposite directions leads to an efficient separation that can be improved further by increasing the number of periodic cavities. A way to quantify this is to calculate the probability that a large particle with an average positive velocity $v>0$ reaches the "wrong" collector, i.e., the left boundary of the device placed at $x=n L$, where $n$ is a negative integer (see Fig. 3). This probability is $P(x<$ $n L, t)=0.5+0.5 \operatorname{erf}\left[(n L-v t) / \sqrt{4 D_{\text {eff }} t}\right]$ where we have assumed that the process can be described as a drivendiffusion process with an average velocity $v$ and an effective diffusion coefficient $D_{\text {eff }}$. This probability peaks in time at $t_{\max }=-n / v$, and its maximum value is at $P\left(x<n L, t_{\max }\right)=0.5+0.5 \operatorname{erf}\left(-\sqrt{v|n| / D_{\text {eff }}}\right)$. We use 


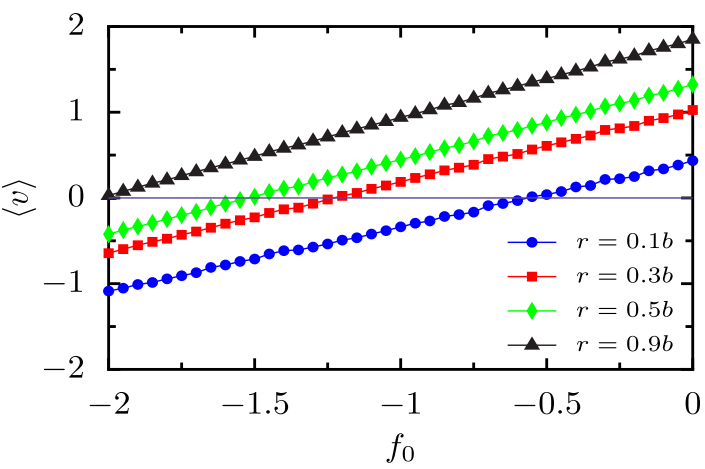

FIG. 4 (color online). Average current $v s$ the value of the static force $f_{0}$ in the adiabatic limit $(\Omega=\pi / 10)$ for particles of different radii, for the channel plotted in Fig. 1 with $F_{0}=20$. In all cases the velocity increases linearly with $f_{0}$. As $f_{0}$ gets more negative, the particles invert their velocity and start to move to the left. The critical force that determines this velocity inversion depends on the radius $r$ and gets progressively larger (in absolute value) for larger radii.

this maximum probability as a measure of the purity of the sample collected at the end of the device. For typical values of the velocity $v \sim 0.2$ (cf. Fig. 4 ) and $D_{\text {eff }} \lesssim 1$, one can achieve $99.9968 \%$ of purity after only 40 periods.

Note, that the entropic splitting effect can be used for separation of DNA fragments of different size using typical values as for DNA electrophoresis. The DNA fragments are considered as randomly coiled polymers with radius $R=d\left(M / M_{0}\right)^{1 / 2}$ where $M$ is the number of base pairs, $d=100 \mathrm{~nm}$ is the Kuhn length, and $M_{0}=300$ is the number of base pairs per Kuhn length [11]. Assuming Stokes' law and the Sutherland-Einstein relation, (i) the friction coefficient for the dynamics of such a DNA fragment in water is $\eta \approx 2 \times 10^{-9} \mathrm{~kg} / \mathrm{s} \sqrt{M / M_{0}}$ and (ii) the diffusion coefficient is $D \approx 2 \times 10^{-8} \mathrm{~cm}^{2} / \mathrm{s} \sqrt{M_{0} / M}$.

For a device with $L=3 \mu \mathrm{m}$ and the same geometry and parameters as in Fig. 1, typical values of the velocities are $L / \tau=D_{0} / L \sim 0.9 \mu \mathrm{m} / \mathrm{s}$, achievable by electrophoresis with an electric field of $7 \mathrm{~V} / \mathrm{cm}$. For DNA chains of $R \sim 250 \mathrm{~nm}$ differing in radius by $\sim 25 \%$, a purity of separation of $99.997 \%$ results after 40 periods. These values compare favorably with commonly used techniques. Much larger velocities and efficiencies are possible with smaller DNA chains, or nanosized particles.

The effectiveness of the entropic splitting can be tuned by choosing the geometry of the channel. In particular, the entropic splitting effect becomes more important upon increasing the asymmetry of the walls, the slopes of the channel or by decreasing the bottleneck width $b$. Altering the design of the channel geometry it is feasible to separate particles of very similar radii, e.g., by choosing $b$ close to $r$. Yet another advantage of this set up is that it works in a time-continuous mode.
In summary, we have presented a novel, purely entropic particle splitting mechanism which is able to separate particles of different sizes. The mechanism is based on the presence of an entropic rectification of fluctuations caused by the asymmetric form of the channel. This rectification may overcome the effect of an applied force by reversing the motion of the particles. The mechanism is very efficient and can be controlled by tuning the geometric parameters of the channel leading to both different velocities and directions. This idea could be implemented in constrained structures with narrow channels and pores where entropic effects are important.

This work has been supported by the Icrea Academia Program, by the MICINN of the Spanish government through the I3 Program and Grant No. FIS2008-01299, by the Max Planck society, the Volkswagen foundation Project No. I/83902 and the German excellence cluster "Nanosystems Initiative Munich" (NIM).

*dreguera@ub.edu

[1] R.G. Harrison et al., Bioseparations Science and Engineering (Oxford University Press, Oxford, 2003).

[2] K. D. Dorfman, Rev. Mod. Phys. 82, 2903 (2010).

[3] W. D. Volkmuth and R. H. Austin, Nature (London) 358, 600 (1992).

[4] G. W. Slater et al., Electrophoresis 23, 3791 (2002).

[5] M. P. MacDonald, G. C. Spalding, and K. Dholakia, Nature (London) 426, 421 (2003).

[6] J. Rousselet et al., Nature (London) 370, 446 (1994).

[7] L. P. Faucheux and A. Libchaber, J. Chem. Soc., Faraday Trans. 91, 3163 (1995).

[8] J.S. Bader et al., Proc. Natl. Acad. Sci. U.S.A. 96, 13165 (1999).

[9] C. Kettner et al., Phys. Rev. E 61, 312 (2000); S. Matthias and F. Müller, Nature (London) 424, 53 (2003).

[10] K. Loutherback et al., Phys. Rev. Lett. 102, 045301 (2009).

[11] T. A. J. Duke and R. H. Austin, Phys. Rev. Lett. 80, 1552 (1998).

[12] R. Eichhorn et al., Soft Matter 6, 1858 (2010).

[13] P. Hänggi and F. Marchesoni, Rev. Mod. Phys. 81, 387 (2009).

[14] G. Schmid et al., Adv. Solid State Phys. 48, 317 (2009).

[15] I. D. Kosinska et al., Phys. Rev. E 77, 031131 (2008).

[16] D. Reguera and J.M. Rubi, Chem. Phys. 375, 518 (2010).

[17] V. Yu. Zitserman et al., J. Chem. Phys. 135, 121102 (2011).

[18] D. Reguera and J. M. Rubi, Phys. Rev. E 64, 061106 (2001).

[19] D. Reguera et al., Phys. Rev. Lett. 96, 130603 (2006).

[20] B.Q. Ai and L.G. Liu, Phys. Rev. E 74, 051114 (2006).

[21] P. S. Burada et al., Phys. Rev. E 75, 051111 (2007).

[22] P. S. Burada et al., Phys. Rev. Lett. 101, 130602 (2008). 
[23] P. S. Burada et al., Europhys. Lett. 87, 50003 (2009).

[24] M. H. Jacobs, Diffusion Processes (Springer, New York, 1967).

[25] R. Zwanzig, J. Phys. Chem. 96, 3926 (1992).

[26] N. A. Fuchs, The Mechanics of Aerosols (Pergamon Press, Oxford, 1964), Section III.17 therein.
[27] R. L. Stratonovich, Radiotekh. Elektron. (Moscow) 3, 497 (1958).

[28] P. Hänggi, P. Talkner, and M. Borkovec, Rev. Mod. Phys. 62, 251 (1990).

[29] P. Reimann et al., Phys. Rev. Lett. 87, 010602 (2001); P. Reimann et al., Phys. Rev. E 65, 031104 (2002). 